\title{
Degradabilidad ruminal de forrajes y residuos de cosecha en bovinos Brown Swiss
}

\author{
Ruminal degradability of forage and crop residues in Brown Swiss cattle
}

José L. Contreras P. ${ }^{1,4}$, Margoreth A. Matos Z. ${ }^{1}$, Erika Felipe C. ${ }^{1}$, Alfonso G. Cordero F. ${ }^{2}$, Yola Ramos Espinoza ${ }^{3}$

\section{Resumen}

\begin{abstract}
El objetivo del trabajo fue determinar la degradabilidad de asociación de forrajes y de residuos de cosecha comúnmente utilizados en la Región de Huancavelica en la alimentación de bovinos. Se evaluaron las asociaciones trébol blanco-ryegrass italiano y el trébol rojo-dactylis, y los residuos de cosecha: maíz chala, paja de avena, arveja, habas y quinua. Para la determinación de la degradabilidad de la materia seca (MS), proteína cruda (PC) y fibra detergente neutra (FDN) se utilizaron tres bovinos Brown Swiss, fistulados en el rumen. Los forrajes y los residuos de cosecha fueron colocados en sacos de nylon e incubados por 4, 8, 12, 24, 48, 72 y 96 horas. El tiempo cero $\left(\mathrm{t}_{0}\right)$ fue utilizado para el cálculo de la fracción soluble. Las degradabilidades de la MS, PC y FDN fueron influenciadas por los forrajes y residuos de cosecha $(\mathrm{p}<0.05)$. La mayor degradabilidad potencial de la MS y PC, y degradabilidad efectiva de la MS, PC y FDN le confieren al trébol blanco-ryegrass italiano un buen potencial nutritivo para los rumiantes. La tasa constante $(\mathrm{C})$ de degradación, degradabilidad potencial y degradabilidad efectiva de la PC permite considerar a los residuos de arveja, habas y quinua como recursos alimenticios de alta calidad nutricional. Por el contrario, la degradabilidad efectiva de la PC a la tasa de pasaje de 5\%/h indica que el maíz chala y la paja de avena son alimentos de mediana calidad.
\end{abstract}

Palabras clave: degradabilidad in situ; parámetros cinéticos; quinua; paja de avena

\footnotetext{
${ }^{1}$ Proyecto de FOCAM, Laboratorio de Nutrición Animal y Evaluación de Alimentos, Facultad de Ciencias de Ingeniería, Universidad Nacional de Huancavelica, Perú

${ }^{2}$ Facultad de Ciencias de Ingeniería, Universidad Nacional de Huancavelica, Perú

${ }^{3}$ Departamento de Zootecnia, Facultad de Ciencias de Ingeniería, Universidad Nacional de Huancavelica, Perú

${ }^{4}$ E-mail: joselcpunh123@hotmail.com
}

Recibido: 9 de octubre de 2018

Aceptado para publicación: 24 de junio de 2019 
The aim of this study was to determine the degradability of the association of forages and crop residues commonly used to feed cattle in the Huancavelica Region. The forage associations were white clover-Italian ryegrass and red clover-dactylis, while the harvest residues were maize, oat straw, peas, beans and quinoa. For the determination of the degradability of dry matter (DM), crude protein (CP) and neutral detergent fibre (NDF), three rumen fistulated Brown Swiss cattle were used. Samples of forages and harvest residues were placed in nylon bags and incubated for 4, 8, 12, 24, 48, 72 and 96 hours. Time zero $\left(\mathrm{t}_{0}\right)$ was used for the calculation of the soluble fraction. The DM, CP and NDF degradability were influenced by forages and crop residues $(p<0.05)$. The greater potential degradability of the DM and CP, and effective degradability of the DM, CP and NDF give the white clover-Italian ryegrass a good nutritional potential for ruminants. The constant rate $(C)$ of degradation, potential degradability and effective degradability of the $\mathrm{CP}$ allows to consider the residues of peas, beans and quinoa as food resources of high nutritional quality. On the contrary, the effective degradability of the $\mathrm{PC}$ at the passage rate of $5 \% / \mathrm{h}$ indicates that maize and oat straw are medium quality foods.

Key words: in situ degradability; kinetic parameters; quinoa; oat straw

\section{INTRODUCCIÓN}

La alfalfa y el trébol solo o asociado con gramíneas componen gran parte de la alimentación de los rumiantes en la sierra del Perú, siendo utilizadas como principales o únicos alimentos voluminosos o groseros en la dieta, además de los residuos de cosecha. Estos forrajes presentan gran variabilidad fisicoquímica y poseen elevada proporción de pared celular (fibra detergente neutra). Esta fracción presenta, de manera general, lenta e incompleta digestión, ocupando espacio en el tracto gastrointestinal (Mertens, 1996) y es la principal responsable por la variación en el consumo y la digestión de los alimentos (Van Soest, 1994; Mertens, 1996). En este contexto, el conocimiento del comportamiento de los alimentos voluminosos durante el proceso de digestión es importante para la determinación del nivel adecuado de su participación en las dietas de los rumiantes. El conocimiento del valor nutritivo de los alimentos que componen la dieta de los animales puede permitir su adecuación con el fin de optimizar el desempeño productivo y disminuir los costos de producción (Cabral et al., 2005).
Para la caracterización de la degradabilidad de los alimentos, la Agricultural and Food Research Council - AFRC (1995) adoptó la técnica de degradación in situ como un método estándar, que produce resultados compatibles con aquellos obtenidos por la técnica in vivo. La primera técnica permite el contacto del alimento evaluado con el ambiente ruminal, siendo la mejor forma de simulación de este medio, pese a que el alimento no está sujeto a todos los eventos digestivos, como la masticación, rumia y pasaje de partículas por el tracto digestivo. La técnica in situ proporciona parámetros cinéticos, relacionados a la degradabilidad ruminal de los nutrientes, difíciles de obtener por otros métodos (in vitro) (Molina et al., 2003).

El conocimiento de las tasas de degradación y el flujo de los alimentos proporcionan datos para un balance más eficiente de las raciones para los rumiantes; sin embargo, bajo las condiciones de la sierra peruana, han sido escasos o nulos los estudios dirigidos a caracterizar la degradación in situ de los nutrientes de forrajes y residuos agrícolas. Teniendo en cuenta estas consideraciones, en la presente investigación se determinó la 
degradabilidad in situ de la materia seca (MS), proteína cruda (PC) y fibra detergente neutra (FDN) de los forrajes asociados trébol blanco-ryegrass italiano (Trifolium repens L - Lolium multiflorum Lam) y trébol rojodactylis (Trifolium pratense L - Dactylis glomerata L), y de los residuos de cosecha maíz chala (Zea mays L), paja de avena (Avena sativa $\mathrm{L}$ ), arveja (Pisum sativum), habas (Vicia faba) y quinua (Chenopodium quinoa).

\section{MATERIALES y Métodos}

\section{Ubicación del Estudio}

La incubación ruminal fue realizada en las instalaciones de la Escuela Académico Profesional de Zootecnia (EAPZ) de la Facultad de Ciencias de Ingeniería, perteneciente a la Universidad Nacional de Huancavelica (UNH), ubicada en el distrito, provincia y región de Huancavelica. La zona se encuentra a $3704 \mathrm{~m}$ sobre el nivel del mar.

\section{Animales y Tratamientos}

Se utilizaron tres toros Brown Swiss, con cánula ruminal permanente, con edades entre 3 y 3.5 años y peso medio de $540 \mathrm{~kg}$. Se tuvo un periodo de adaptación de 12 días, donde los animales fueron alimentados con una dieta de partes iguales de alfalfa (Medicago sativa) y heno de avena (Avena sativa $\mathrm{L}$ ), suministrada a las 08:00 y 17:00 horas, disponiendo de agua y sal mineral a voluntad. La proporción de la mezcla permaneció constante durante todo el periodo experimental.

Se trabajó con seis tratamientos: $\mathrm{T} 1=$ trébol blanco-ryegrass italiano (Trifolium repens L- Lolium multiflorum Lam); T2 = trebol rojo-dactylis ( Trifolium pratense L Dactylis glomerata L); T3 = maíz chala (Zea mays $\mathrm{L}$ ); $\mathrm{T} 4$ = paja de avena (Avena sativa L); T5 = arveja (Pisum sativum); T6 = habas $($ Vicia faba $) ; \mathrm{T} 7=$ quinua $($ Cheno- podium quinoa). La edad estimada de los forrajes de T1 y T2 fue de 12 semanas de crecimiento y la de los residuos de cosecha de 20 semanas.

\section{Muestras}

Los forrajes fueron obtenidos directamente de las propiedades rurales de las provincias de Tayacaja, Acobamba, Huaytara y Huancavelica de la región de Huancavelica; considerándose dos épocas del año: noviembre-marzo (lluvias) y abril-agosto (seca). Los residuos de cosecha se adquirieron de productores agropecuarios de la región. Para el muestreo de los forrajes se utilizó la técnica no probabilística de tipo intencionada. Se recolectó por conveniencia los forrajes de las áreas de producción (Lagares y Puerto, 2001). Las muestras fueron secadas en estufa a $65^{\circ} \mathrm{C}$ durante 72 horas, para luego ser molidas en molino tipo Willy en tamiz de $3 \mathrm{~mm}$.

\section{Degradabilidad Ruminal}

Se utilizaron bolsas de nylon (Ankom) de $7 \times 12 \mathrm{~cm}$, con poros de abertura de aproximadamente $50 \mu \mathrm{m}$. En cada bolsa fue colocada una muestra de $5 \mathrm{~g}$ (base en la MS). Todas las muestras fueron incubadas en duplicado y en un mismo momento en el rumen. Para la incubación, las bolsas fueron adheridas a una cadena de acero inoxidable que estaba suspendida por un hilo de nylon en la cánula. Los tiempos de incubación fueron 4 , $8,12,24,48,72$ y 96 horas. Las bolsas correspondientes al tiempo cero horas $\left(\mathrm{t}_{0}\right)$ no fueron incubados, pero fueron inmersas en agua a $39^{\circ} \mathrm{C}$ por 15 minutos y secadas en estufa de ventilación forzada a $60^{\circ} \mathrm{C}$ por 24 horas.

Las bolsas de nylon, una vez retiradas del rumen fueron colocadas en un balde con agua fría durante 5 minutos para interrumpir la actividad microbiana. Luego fueron lavadas en agua corriente para remover las partículas adheridas a la superficie externa, hasta obtener agua clara, sin materiales en suspensión. Posteriormente, las bolsas fueron seca- 
das en estufa de ventilación forzada a $60^{\circ} \mathrm{C}$ por 24 horas. La desaparición in situ de la MS (ISDMD, por sus siglas en inglés: in situ dry matter degradability) se determinó utilizando la siguiente fórmula: ([Muestra incubada, g - Residuo, g]/Muestra incubada, g)*100. Una fracción de cada muestra de residuo fue utilizada para la determinación de la proteína cruda (PC: Kjeldahl) (Silva y Queiroz, 2002) y FDN, usando un analizador de fibra Ankom 200 (Ankom Technology Corp., Macedon, NY).

Los parámetros de cinética de degradación ruminal de la MS, PC y FDN fueron calculados por la ecuación descrita por Orskov y McDonald (1979), donde Dt = $\mathrm{a}+\mathrm{b} *(1$-expct $), y \mathrm{Dt}=$ fracción degradada en el tiempo $\mathrm{t}(\%) ;(\mathrm{a})=$ fracción soluble $(\%)$; (b) = fracción insoluble potencialmente degradable $(\%) ;(c)=$ tasa de degradación de la fracción (b) $(\% /$ hora); $t$ = tiempo( horas); exp = base de los logaritmos naturales; $\langle a »+\langle\langle\mathrm{b} » \leq 100$.

Los parámetros no lineares (a), (b) y (c) fueron estimados con el aplicativo Solver de Microsoft Excel. La degradabilidad efectiva (DE) de la MS, PC y FDN en el rumen fue calculada por la ecuación de Orskov y McDonald (1979), donde DE $=\mathrm{a}+[(\mathrm{b} \times \mathrm{c}) /$ $(\mathrm{c}+\mathrm{k})]$, y que $\mathrm{k}=$ es la tasa estimada de pasaje de las partículas en el rumen (los demás parámetros fueron descritos en la ecuación anterior).

La DE de la MS, PC y FD fueron estimadas para cada alimento, considerándose la tasa de pasaje de sólidos por el rumen de 2,5 y $8 \% / h$, que puede ser atribuido a un nivel de consumo alimenticio bajo, medio y alto, según AFRC (1995).

\section{Diseño Experimental y Análisis Estadís- tico}

Se utilizó el diseño de bloques completamente al azar (3 animales) con arreglo factorial de 7 x 8 (forrajes y residuos, tiempos), con el modelo lineal: $\mathrm{Yijk}=\mu+\mathrm{Fi}+\mathrm{Tj}+$
(FT)ij+eijk, donde Yijk = variable respuesta (parámetros de la degradación in situ), $\mu=$ Media general, $\mathrm{Fi}=$ efecto de los forrajes y residuos $(\mathrm{i}=1,2,3,4,5,6,7), \mathrm{Tj}=$ efecto de los tiempos de incubación ruminal $(\mathrm{j}=1,2,3$, $4,5,6,7,8),(\mathrm{FT}) \mathrm{ij}=$ efecto de la interacción entre los factores $\mathrm{F}$ y $\mathrm{T}$, y eijk = variable aleatoria normal e independientemente distribuida con una media $\varnothing$ y variancia $\sigma \mathrm{e}^{2}$.

El análisis de los datos de degradación in situ para los alimentos fueron realizados adoptándose el procedimiento GLM del SAS v. 9.2 (2009). La comparación de medias fue mediante la prueba de Tukey, con nivel de significación de $\mathrm{p}<0.05$ de probabilidad.

\section{Resultados y Discusión}

\section{Desaparición de los Nutrientes}

La desaparición media en el tiempo $\mathrm{t}_{0} \mathrm{y}$ en los tiempos de incubación ruminal (horas) en bovinos Brown Swiss de la materia seca (MS), proteína cruda (PC) y fibra detergente neutra (FDN) de los forrajes (trébol blancoreygrass italiano y trébol rojo-dactylis y de los residuos de cosecha (maíz chala fresco, paja de avena, arveja, habas y quinua se presentan en el Cuadro 1.

Las fracciones solubles en el $\mathrm{t}_{0}$ de la MS oscilaron entre 8.64 y $9.13 \%$ para los forrajes. Pariona (2018) obtuvo medias de $19.20,13.17$ y $19.33 \%$ para la alfalfa, alfalfa-trébol rojo y alfalfa-dactylis, respectivamente, mientras que Molina et al. (2003) encontraron valores medios en el $\mathrm{t}_{0}$ entre 15.14 y $23.01 \%$ para ensilados de sorgo. Estos altos valores de solubilidad en $\mathrm{t}_{0}$ podrían deberse a que en moliendas muy finas el material puede salir fácil y rápidamente por los poros de la bolsa de incubación; sin embargo, Michalet y Ould-Bah (1992) indican que las partículas que escapan de las bolsas y que no son degradadas no afectarían la degradabilidad final del material incubado. Por otro lado, la desaparición media de la MS en $\mathrm{t}_{0}$ 
Cuadro 2. Fracciones solubles (A), insolubles potencialmente degradables (B), indigestible (I), tasa de degradación constante de la fracción B (C), degradabilidades potenciales (DP) y efectivas (DE) de la materia seca, proteína cruda y fibra detergente neutra de forrajes y residuos de cosecha.

\begin{tabular}{|c|c|c|c|c|c|c|c|c|}
\hline & \multicolumn{3}{|c|}{ Fracciones (\%) } & \multirow{2}{*}{$\begin{array}{c}\mathrm{C} \\
(\% / \mathrm{h})\end{array}$} & \multirow{2}{*}{$\begin{array}{l}\mathrm{DP} \\
(\%)\end{array}$} & \multicolumn{3}{|c|}{$\begin{array}{l}\text { Degradabilidades efectivas } \\
\text { (\%) a tasas de flujo de }\end{array}$} \\
\hline & $\mathrm{A}$ & $\mathrm{B}$ & $\mathrm{I}$ & & & $2 \% / \mathrm{h}$ & $5 \% / \mathrm{h}$ & $8 \% / \mathrm{h}$ \\
\hline & \multicolumn{8}{|c|}{ Materia seca } \\
\hline \multicolumn{9}{|l|}{ Forrajes } \\
\hline $\mathrm{TB}^{\mathrm{R} E^{1}}$ & $8.60^{\mathrm{bc}}$ & $62.84^{\mathrm{bc}}$ & 28.56 & $1.33^{\mathrm{c}}$ & $71.44^{\mathrm{ab}}$ & $38.22^{c}$ & $24.07^{\mathrm{c}}$ & $19.03^{c}$ \\
\hline TR-DA ${ }^{2}$ & $9.46^{\mathrm{b}}$ & $54.13^{\mathrm{d}}$ & 36.41 & $2.00^{\mathrm{b}}$ & $63.59^{c}$ & $31.70^{\mathrm{d}}$ & $21.89^{d}$ & $18.05^{\mathrm{c}}$ \\
\hline \multicolumn{9}{|l|}{ Residuos } \\
\hline $\mathrm{MCH}^{3}$ & $19.65^{\mathrm{a}}$ & $44.88^{\mathrm{e}}$ & 35.47 & $2.67^{\mathrm{a}}$ & $64.53^{\mathrm{c}}$ & $44.05^{\mathrm{a}}$ & $34.38^{\mathrm{a}}$ & $30.14^{\mathrm{a}}$ \\
\hline $\mathrm{PA}^{4}$ & $6.56^{\mathrm{d}}$ & $63.69^{\mathrm{abc}}$ & 29.75 & $1.00^{\mathrm{c}}$ & $70.25^{\mathrm{bc}}$ & $26.17^{\mathrm{e}}$ & $17.15^{\mathrm{f}}$ & $14.30^{\mathrm{d}}$ \\
\hline $\mathrm{AR}^{5}$ & $7.15^{\mathrm{cd}}$ & $67.30^{\mathrm{ab}}$ & 25.55 & $1.33^{\mathrm{c}}$ & $74.45^{\mathrm{ab}}$ & $30.69^{d}$ & $19.14^{\mathrm{e}}$ & $15.08^{\mathrm{d}}$ \\
\hline $\mathrm{HA}^{6}$ & $7.83^{\mathrm{cd}}$ & $61.00^{c}$ & 31.17 & $2.33^{\mathrm{ab}}$ & $68.83^{\mathrm{bc}}$ & $40.72^{\mathrm{b}}$ & $28.43^{\mathrm{b}}$ & $23.16^{\mathrm{b}}$ \\
\hline $\mathrm{QU}^{7}$ & $7.52^{\mathrm{cd}}$ & $69.29^{\mathrm{a}}$ & 23.19 & $1.33^{\mathrm{c}}$ & $76.81^{\mathrm{a}}$ & $31.71^{\mathrm{d}}$ & $21.47^{\mathrm{d}}$ & $17.79^{\mathrm{c}}$ \\
\hline & \multicolumn{8}{|c|}{ Proteína cruda } \\
\hline \multicolumn{9}{|l|}{ Forrajes } \\
\hline TB-RE ${ }^{1}$ & $24.50^{\mathrm{ab}}$ & $49.22^{\mathrm{c}}$ & 26.28 & $1.67^{\mathrm{c}}$ & 26.28 & $49.98^{\mathrm{d}}$ & $38.46^{\mathrm{d}}$ & $38.46^{\mathrm{d}}$ \\
\hline TR-DA ${ }^{2}$ & $11.96^{\mathrm{d}}$ & $56.34^{\mathrm{abc}}$ & 31.70 & $2.33^{c}$ & 31.70 & $39.32^{\mathrm{e}}$ & $29.98^{\mathrm{e}}$ & $29.98^{\mathrm{e}}$ \\
\hline \multicolumn{9}{|l|}{ Residuos } \\
\hline $\mathrm{MCH}^{3}$ & $11.40^{\mathrm{d}}$ & $59.72^{\mathrm{a}}$ & 28.88 & $1.67^{\mathrm{c}}$ & 28.88 & $46.05^{\mathrm{d}}$ & $31.89^{\mathrm{e}}$ & $31.89^{\mathrm{e}}$ \\
\hline $\mathrm{PA}^{4}$ & $15.41^{\mathrm{cd}}$ & $51.42^{\mathrm{bc}}$ & 33.17 & $2.00^{c}$ & 33.17 & $47.39^{\mathrm{d}}$ & $36.63^{\mathrm{d}}$ & $36.63^{\mathrm{d}}$ \\
\hline $\mathrm{AR}^{5}$ & $16.36^{\mathrm{cd}}$ & $58.67^{\mathrm{ab}}$ & 24.97 & $5.67^{b}$ & 24.97 & $58.89^{\mathrm{c}}$ & $44.63^{\mathrm{c}}$ & $44.63^{\mathrm{c}}$ \\
\hline $\mathrm{HA}^{6}$ & $20.18^{\mathrm{cb}}$ & $60.07^{\mathrm{a}}$ & 19.75 & $4.67^{b}$ & 19.75 & $66.82^{\mathrm{b}}$ & $57.21^{\mathrm{b}}$ & $57.21^{\mathrm{b}}$ \\
\hline $\mathrm{QU}^{7}$ & $27.06^{\mathrm{a}}$ & $50.79^{c}$ & 22.15 & $7.66^{\mathrm{a}}$ & 22.15 & $71.15^{\mathrm{a}}$ & $62.85^{\mathrm{a}}$ & $62.85^{\mathrm{a}}$ \\
\hline
\end{tabular}

Fibra detergente neutro

Forrajes

\begin{tabular}{ccccccccc} 
TB-RE & $11.51^{\mathrm{b}}$ & $53.57^{\mathrm{bc}}$ & 34.92 & $1.67^{\mathrm{cd}}$ & $65.08^{\mathrm{c}}$ & $33.37^{\mathrm{c}}$ & $26.33^{\mathrm{c}}$ & $22.02^{\mathrm{c}}$ \\
$\begin{array}{c}\text { TR-DA } \\
\text { Residuos }\end{array}$ & $16.87^{\mathrm{a}}$ & $53.98^{\mathrm{bc}}$ & 29.15 & $1.00^{\mathrm{e}}$ & $70.85^{\mathrm{a}}$ & $34.57^{\mathrm{c}}$ & $25.74^{\mathrm{cd}}$ & $22.82^{\mathrm{c}}$ \\
$\mathrm{MCH}^{3}$ & $15.60^{\mathrm{a}}$ & $55.59^{\mathrm{bc}}$ & 28.81 & $2.00^{\mathrm{c}}$ & $71.19^{\mathrm{a}}$ & $43.34^{\mathrm{b}}$ & $32.17^{\mathrm{b}}$ & $27.78^{\mathrm{b}}$ \\
$\mathrm{PA}^{4}$ & $11.02^{\mathrm{b}}$ & $62.69^{\mathrm{a}}$ & 26.29 & $1.33^{\mathrm{de}}$ & $73.71^{\mathrm{a}}$ & $32.55^{\mathrm{c}}$ & $22.23^{\mathrm{d}}$ & $18.42^{\mathrm{d}}$ \\
$\mathrm{AR}^{5}$ & $13.24^{\mathrm{b}}$ & $51.56^{\mathrm{c}}$ & 35.20 & $2.67^{\mathrm{b}}$ & $64.80^{\mathrm{c}}$ & $43.13^{\mathrm{b}}$ & $30.14^{\mathrm{b}}$ & $24.34^{\mathrm{c}}$ \\
$\mathrm{HA}^{6}$ & $12.14^{\mathrm{b}}$ & $57.54^{\mathrm{ab}}$ & 30.32 & $3.00^{\mathrm{b}}$ & $69.68^{\mathrm{ab}}$ & $50.22^{\mathrm{a}}$ & $37.17^{\mathrm{a}}$ & $31.28^{\mathrm{a}}$ \\
$\mathrm{QU}^{7}$ & $7.48^{\mathrm{c}}$ & $58.05^{\mathrm{ab}}$ & 34.47 & $3.67^{\mathrm{a}}$ & $65.53^{\mathrm{bc}}$ & $52.75^{\mathrm{a}}$ & $40.09^{\mathrm{a}}$ & $33.80^{\mathrm{a}}$ \\
\hline
\end{tabular}

${ }^{1}$ trébol blanco-ryegrass italiano, ${ }^{2}$ trébol rojo-dactylis, ${ }^{3}$ maíz chala, ${ }^{4}$ paja de avena, ${ }^{5}$ arveja, ${ }^{6}$ habas,

${ }^{7}$ quinua

Medias con las mismas letras en columnas no difieren significativamente entre sí 
para los residuos de cosecha varió entre 5.15 a $16.50 \%$.

Para el trébol blanco y trébol rojodactylis se observó una estabilización de las medias de desaparición de la MS a partir de las 72 horas de incubación, no habiendo diferencia con los valores a las 96 horas. Comportamiento similar ocurrió con los residuos, excepto para la arveja; hecho que demuestra que la incubación por 96 horas es suficiente para alcanzar los valores máximos de desaparecimiento de la MS, es decir, la asíntota. Así mismo, no se observó diferencia significativa en el desaparecimiento de la MS entre los dos grupos de forrajes en cada uno de los tiempos de incubación.

En el caso de los residuos, la desaparición de la MS del maíz chala fue mayor a partir de las 4 horas de incubación ruminal con relación a la paja de avena (25.02 vs. $8.08 \%$; $\mathrm{p}<0.05$ ); en tanto que no hubo diferencias significativas entre el maíz chala y las habas para la desaparición media de la MS a las 72 horas, ni entre todos los residuos de cosecha a las 96 horas, excepto para la paja de avena, cuya media fue significativamente menor $(8.76 \% ; \mathrm{p}<0.05)$.

Los forrajes trébol rojo-dactylis y trébol blanco-ryegrass presentaron valores de solubilidad para la PC para el tiempo cero entre 10.93 y $22.37 \%$, cifras que representan la porción del nitrógeno soluble o partículas pequeñas lo suficientemente molidas que salen por los poros de las bolsas de incubación (Molina et al., 2003). Se observó una estabilización de las medias de desaparición de la PC a partir de las 48 horas del trébol blancoryegrass italiano y trébol rojo-dactylis, pues no mostraron diferencias significativas con las 72 y 96 horas de incubación. Estos resultados indican que incubaciones por 96 horas son apropiadas para lograr los valores máximos de desaparición de la PC de estos forrajes. Pariona (2018) observó 96.87, 90.07 y $88.48 \%$ de desaparición de la PC a las 96 horas de incubación en alfalfa, alfalfa-trébol rojo y alfalfa-dactylis, y con estabilización a partir de las 48 horas de incubación. Las fracciones solubles en el tiempo cero de la PC de los residuos variaron entre 11.90 y $22.64 \%$.

En el caso de los residuos, se observó la estabilización en la desaparición de la PC en el maíz chala, arveja, habas y quinua a partir de las 48 horas de incubación ruminal, sin diferencia significativa con los valores a las 72 y 96 horas de incubación. La estabilización se presentó en la paja de avena a partir de las 72 horas, lo que confirma que los máximos valores de desaparición de la PC se pueden observar a las 96 horas. Los residuos de arveja, habas y quinua presentaron los mayores desapariciones de la PC a partir de las 4 horas de incubación en comparación con los residuos de maíz chala y paja de avena, pero sin diferencia significativa en los resultados a las 96 horas de incubación. Los menores valores, aunque no significativos, que presentaron los residuos de maíz chala y paja de avena a las 96 horas de incubación (59.99 y $63.70 \%$, respectivamente), podrían haberse debido al elevado coeficiente de variación observado para la desaparición de la PC $(12.0 \%)$, lo cual, a su vez, sería una consecuencia de la falta de homogeneidad de las muestras incubadas en el rumen.

Con relación a la FDN, no se observaron diferencias significativas entre forrajes. Ambos grupos encontraron la estabilización a partir de las 72 horas de incubación. En los residuos de cosecha no se verificaron diferencias significativas en la desaparición de la FDN en los tiempos de incubación de 4, 8, 12 y 24 horas de incubación. Se observó estabilización de la desaparición de la FDN a partir de las 72 horas de incubación, excepto para el maíz chala.

\section{Degradabilidad de la Materia Seca, Pro- teína Cruda y Fibra Detergente Neutra}

El análisis de variancia mostró efectos significativos $(\mathrm{p}<0.05)$ de los forrajes y de los residuos de cosecha sobre los coeficientes (A) (fracción soluble), (B) (fracción insoluble pero potencialmente degradable), (C) 
(tasa de degradación), $\mathrm{A}+\mathrm{B}$ (degradabilidad potencial) y DE (degradabilidad efectiva) para las tasas de flujos de 2,5 y $8 \% / \mathrm{h}$ de la MS. Las asociaciones de trébol blancoryegrass italiano y trébol rojo-dactylis no mostraron diferencias significativas en la fracción soluble (A) de la MS (Cuadro 2), con tasas de degradación (C) bajas y diferentes $(1.33$ y $2.00 \% / h)$, hecho que pudiera atribuirse al elevado contenido de FDN $(45.15 \%$ en promedio), semejante a lo referenciado por Martins et al.(1999) para el ensilado de maíz.

Las tasas de degradaciones observadas en el presente trabajo son inferiores a los resultados obtenidos por Pariona (2018), que trabajó con las asociaciones de alfalfa-trébol rojo $(3.06 \% / \mathrm{h})$ y alfalfa-dactylis $(7.06 \% / \mathrm{h})$. Factores como el contenido de MS de la especie, el tipo de fermentación y el contenido de carbohidratos solubles pueden contribuir para obtener diferentes tasas de degradación ruminal (Martins et al., 1999). Entre los residuos de cosecha, el maíz chala destacó en la fracción soluble (A) $(19.65 \%)$ con tasa de degradación de $2.67 \% / \mathrm{h}$; sin embargo, este valor es inferior a la tasa de degradación de $12.57 \% / \mathrm{h}$ obtenido para el ensilado de maíz chala en ensayos de degradabilidad con alpacas (Cordero et al., 2018). En este estudio, la fracción soluble (A) fue $21.74 \%$, en tanto que esta fracción varió entre $6.56 \mathrm{y}$ $7.15 \%$ para los demás residuos de cosecha, con tasas de degradación de 1.00 a $2.33 \% / \mathrm{h}$.

La fracción insoluble (B) del trébol blanco-ryegrass italiano sobresalió en relación al trébol rojo-dactylis y, consecuentemente, presentó mayor degradabilidad potencial de la MS (DPMS) $(71.45 \%)$. Sin embargo, las degradabilidades efectivas de la MS (DEMS) fueron similares y bajas) para la tasa de flujo de $8 \% / \mathrm{h}$ (19.03 vs. 18.05 para estos forrrajes). Cuando se compara la fracción (B) (81.11 y $58.73 \%$ ) y DPMS (91.46 y $79.09 \%$ ) observadas por Pariona (2018) en la alfalfatrébol rojo y alfalfa-dactylis, respectivamente, se nota que ambas variables son superiores respecto al trébol blanco-ryegrass italiano y trébol rojo-dactylis obtenidos en el presente trabajo.
Los valores encontrados para la fracción (B) (44.88), DPMS (64.53\%) y DEMS $(30.14 \%)$ a la tasa de flujo de $8 \% / \mathrm{h}$ para el residuo de maíz chala son inferiores a los valores relatados por Cordero et al. (2018) de $56.36,78.10$ y $56.19 \%$, respectivamente, para el ensilado de maíz chala. Cabral et al. (2005), por otro lado, encontraron el valor de $57.15 \%$ para la fracción (B) de la MS del ensilado de maíz chala, superior al $44.88 \%$ del presente estudio. Estas diferencias pueden ser atribuidas a la diversidad de variedades utilizadas y a su forma de conservación. El maíz chala presentó baja tasa de degradación $(2.67 \% / \mathrm{h})$, siendo considerado como un alimento de baja degradación ruminal (Martins et al., 1999).

Los residuos de paja de avena, arveja, habas y quinua presentaron tasas de degradación que variaron entre 1.00 (paja de avena) y $2.33 \% / \mathrm{h}$ (habas), siendo clasificados como alimentos de baja degradabilidad de la MS. Los valores de la DEMS oscilaron entre 14.30 y $23.16 \%$ y la fracción soluble (A) entre 6.56 y $7.83 \%$. Estos residuos, bajo estos parámetros, son considerados como alimentos de muy lenta tasa de degradación de la MS (Martins et al., 1999).

En el Cuadro 2 se presentan los resultados para los parámetros (A), (B) y (C), las degradabilidades potenciales (DP) y efectivas (DE) de la PC (DEPC) para las tasas de flujo de 2,5 y $8 \% / \mathrm{h}$. Los parámetros cinéticos y degradabilidades de la PC fueron significativamente influenciados $(\mathrm{p}<0.05)$ por los forrajes y los residuos de cosecha. Los valores de DEPC para el trébol blancoryegrass italiano $(38.46 \%)$ y el trébol rojodactylis (29.98\%) fueron inferiores a los encontrados por Pariona (2018) en la alfalfatrébol rojo $(64.48 \%)$ y alfalfa-dactylis (66.29\%) para el flujo de 5\%/h. Los forrajes en estudio presentaron baja degradación ruminal, con valores de 1.67 y $2.33 \% / \mathrm{h}$ para (C), con fracciones solubles de 24.50 y $11.96 \%$, respectivamente. El trébol rojo-dactylis presentó mayor cantidad de PC (13.81\%) que el trébol blanco blanco-ryegrass italiano (5.31\%). 
Cuadro 2. Fracciones solubles (A), insolubles potencialmente degradables (B), indigestible (I), tasa de degradación constante de la fracción B (C), degradabilidades potenciales (DP) y efectivas (DE) de la materia seca, proteína cruda y fibra detergente neutra de forrajes y residuos de cosecha.

\begin{tabular}{|c|c|c|c|c|c|c|c|c|}
\hline & \multicolumn{3}{|c|}{ Fracciones (\%) } & \multirow{2}{*}{$\begin{array}{c}\mathrm{C} \\
(\% / \mathrm{h})\end{array}$} & \multirow{2}{*}{$\begin{array}{l}\text { DP } \\
(\%)\end{array}$} & \multicolumn{3}{|c|}{$\begin{array}{l}\text { Degradabilidades efectivas } \\
(\%) \text { a tasas de flujo de }\end{array}$} \\
\hline & $\mathrm{A}$ & $\mathrm{B}$ & $\mathrm{I}$ & & & $2 \% / \mathrm{h}$ & $5 \% / \mathrm{h}$ & $8 \% / \mathrm{h}$ \\
\hline & \multicolumn{8}{|c|}{ Materia seca } \\
\hline \multicolumn{9}{|l|}{ Forrajes } \\
\hline TB-RE ${ }^{1}$ & $8.60^{\mathrm{bc}}$ & $62.84^{\mathrm{bc}}$ & 28.56 & $1.33^{\mathrm{c}}$ & $71.44^{\mathrm{ab}}$ & $38.22^{\mathrm{c}}$ & $24.07^{\mathrm{c}}$ & $19.03^{c}$ \\
\hline TR-DA ${ }^{2}$ & $9.46^{\mathrm{b}}$ & $54.13^{d}$ & 36.41 & $2.00^{\mathrm{b}}$ & $63.59^{c}$ & $31.70^{\mathrm{d}}$ & $21.89^{d}$ & $18.05^{\mathrm{c}}$ \\
\hline \multicolumn{9}{|l|}{ Residuos } \\
\hline $\mathrm{MCH}^{3}$ & $19.65^{\mathrm{a}}$ & $44.88^{\mathrm{e}}$ & 35.47 & $2.67^{\mathrm{a}}$ & $64.53^{c}$ & $44.05^{\mathrm{a}}$ & $34.38^{\mathrm{a}}$ & $30.14^{\mathrm{a}}$ \\
\hline $\mathrm{PA}^{4}$ & $6.56^{\mathrm{d}}$ & $63.69^{\mathrm{abc}}$ & 29.75 & $1.00^{\mathrm{c}}$ & $70.25^{\mathrm{bc}}$ & $26.17^{\mathrm{e}}$ & $17.15^{\mathrm{f}}$ & $14.30^{\mathrm{d}}$ \\
\hline $\mathrm{AR}^{5}$ & $7.15^{\mathrm{cd}}$ & $67.30^{\mathrm{ab}}$ & 25.55 & $1.33^{\mathrm{c}}$ & $74.45^{\mathrm{ab}}$ & $30.69^{d}$ & $19.14^{\mathrm{e}}$ & $15.08^{\mathrm{d}}$ \\
\hline $\mathrm{HA}^{6}$ & $7.83^{\mathrm{cd}}$ & $61.00^{\mathrm{c}}$ & 31.17 & $2.33^{\mathrm{ab}}$ & $68.83^{b c}$ & $40.72^{b}$ & $28.43^{\mathrm{b}}$ & $23.16^{\mathrm{b}}$ \\
\hline $\mathrm{QU}^{7}$ & $7.52^{\mathrm{cd}}$ & $69.29^{\mathrm{a}}$ & 23.19 & $1.33^{\mathrm{c}}$ & $76.81^{\mathrm{a}}$ & $31.71^{\mathrm{d}}$ & $21.47^{\mathrm{d}}$ & $17.79^{\mathrm{c}}$ \\
\hline & \multicolumn{8}{|c|}{ Proteína cruda } \\
\hline \multicolumn{9}{|l|}{ Forrajes } \\
\hline $\mathrm{TB}^{\mathrm{R} \mathrm{E}^{1}}$ & $24.50^{\mathrm{ab}}$ & $49.22^{c}$ & 26.28 & $1.67^{\mathrm{c}}$ & 26.28 & $49.98^{\mathrm{d}}$ & $38.46^{\mathrm{d}}$ & $38.46^{\mathrm{d}}$ \\
\hline TR-DA ${ }^{2}$ & $11.96^{\mathrm{d}}$ & $56.34^{\mathrm{abc}}$ & 31.70 & $2.33^{c}$ & 31.70 & $39.32^{\mathrm{e}}$ & $29.98^{\mathrm{e}}$ & $29.98^{\mathrm{e}}$ \\
\hline \multicolumn{9}{|l|}{ Residuos } \\
\hline $\mathrm{MCH}^{3}$ & $11.40^{\mathrm{d}}$ & $59.72^{\mathrm{a}}$ & 28.88 & $1.67^{\mathrm{c}}$ & 28.88 & $46.05^{\mathrm{d}}$ & $31.89^{\mathrm{e}}$ & $31.89^{\mathrm{e}}$ \\
\hline $\mathrm{PA}^{4}$ & $15.41^{\mathrm{cd}}$ & $51.42^{\mathrm{bc}}$ & 33.17 & $2.00^{\mathrm{c}}$ & 33.17 & $47.39^{d}$ & $36.63^{d}$ & $36.63^{\mathrm{d}}$ \\
\hline $\mathrm{AR}^{5}$ & $16.36^{\mathrm{cd}}$ & $58.67^{\mathrm{ab}}$ & 24.97 & $5.67^{b}$ & 24.97 & $58.89^{\mathrm{c}}$ & $44.63^{\mathrm{c}}$ & $44.63^{c}$ \\
\hline $\mathrm{HA}^{6}$ & $20.18^{\mathrm{cb}}$ & $60.07^{\mathrm{a}}$ & 19.75 & $4.67^{b}$ & 19.75 & $66.82^{\mathrm{b}}$ & $57.21^{\mathrm{b}}$ & $57.21^{\mathrm{b}}$ \\
\hline \multirow[t]{2}{*}{$\mathrm{QU}^{7}$} & $27.06^{\mathrm{a}}$ & $50.79^{c}$ & 22.15 & $7.66^{\mathrm{a}}$ & 22.15 & $71.15^{\mathrm{a}}$ & $62.85^{\mathrm{a}}$ & $62.85^{\mathrm{a}}$ \\
\hline & \multicolumn{8}{|c|}{ Fibra detergente neutro } \\
\hline \multicolumn{9}{|l|}{ Forrajes } \\
\hline $\mathrm{TB}^{\mathrm{RE}} \mathrm{P}^{1}$ & $11.51^{\mathrm{b}}$ & $53.57^{\mathrm{bc}}$ & 34.92 & $1.67^{\mathrm{cd}}$ & $65.08^{c}$ & $33.37^{\mathrm{c}}$ & $26.33^{\mathrm{c}}$ & $22.02^{\mathrm{c}}$ \\
\hline TR-DA ${ }^{2}$ & $16.87^{\mathrm{a}}$ & $53.98^{\mathrm{bc}}$ & 29.15 & $1.00^{\mathrm{e}}$ & $70.85^{\mathrm{a}}$ & $34.57^{\mathrm{c}}$ & $25.74^{\mathrm{cd}}$ & $22.82^{\mathrm{c}}$ \\
\hline \multicolumn{9}{|l|}{ Residuos } \\
\hline $\mathrm{MCH}^{3}$ & $15.60^{\mathrm{a}}$ & $55.59^{\mathrm{bc}}$ & 28.81 & $2.00^{\mathrm{c}}$ & $71.19^{\mathrm{a}}$ & $43.34^{\mathrm{b}}$ & $32.17^{\mathrm{b}}$ & $27.78^{\mathrm{b}}$ \\
\hline $\mathrm{PA}^{4}$ & $11.02^{\mathrm{b}}$ & $62.69^{\mathrm{a}}$ & 26.29 & $1.33^{\mathrm{de}}$ & $73.71^{\mathrm{a}}$ & $32.55^{\mathrm{c}}$ & $22.23^{\mathrm{d}}$ & $18.42^{\mathrm{d}}$ \\
\hline $\mathrm{AR}^{5}$ & $13.24^{\mathrm{b}}$ & $51.56^{\mathrm{c}}$ & 35.20 & $2.67^{\mathrm{b}}$ & $64.80^{\mathrm{c}}$ & $43.13^{b}$ & $30.14^{\mathrm{b}}$ & $24.34^{\mathrm{c}}$ \\
\hline $\mathrm{HA}^{6}$ & $12.14^{\mathrm{b}}$ & $57.54^{\mathrm{ab}}$ & 30.32 & $3.00^{\mathrm{b}}$ & $69.68^{\mathrm{ab}}$ & $50.22^{\mathrm{a}}$ & $37.17^{\mathrm{a}}$ & $31.28^{\mathrm{a}}$ \\
\hline $\mathrm{QU}^{7}$ & $7.48^{c}$ & $58.05^{\mathrm{ab}}$ & 34.47 & $3.67^{\mathrm{a}}$ & $65.53^{\mathrm{bc}}$ & $52.75^{\mathrm{a}}$ & $40.09^{\mathrm{a}}$ & $33.80^{\mathrm{a}}$ \\
\hline
\end{tabular}

${ }^{1}$ trébol blanco-ryegrass italiano, ${ }^{2}$ trébol rojo-dactylis, ${ }^{3}$ maíz chala, ${ }^{4}$ paja de avena, ${ }^{5}$ arveja, ${ }^{6}$ habas, ${ }^{7}$ quinua Medias con las mismas letras en columnas no difieren significativamente entre sí 
La fracción (A) incluye moléculas en forma de nitrógeno no proteico (urea, aminoácidos libres y péptidos pequeños), las cuales se liberan cuando el alimento llega al rumen y se convierten rápidamente en $\mathrm{N}$ amoniacal. La contribución de este nitrógeno a la producción de proteína microbiana es importante (Klopfenstein et al., 2001); sin embargo, existe un límite por encima del cual la fracción (A) no es fisiológicamente aceptable, ya que no debe superar el $40 \%$ de la DE de la PC (AFRC, 1995). Considerando la DEPC al flujo de las partículas en el rumen de $5 \% / \mathrm{h}$, la asociación trébol blanco-ryegrass italiano presentó la mayor relación (A):DEPC $=63.70 \%((100 * 24.50) / 38.46))$ que la asociación trébol rojo-dactylis $(40.00 \%)$, indicando que la primera asociación de forrajes da lugar a la mayor pérdida de $\mathrm{N}$ en los rumiantes. Pariona (2018), para la asociación alfalfa-dactylis observó las relaciones (A):DEPC de 27.53 y $30.93 \%$ para las tasas de flujo de 5 y $8 \% / h$, respectivamente. Es así que se concluye que leguminosas asociadas con el pasto dactylis permite evitar mayores pérdidas de $\mathrm{N}$ por los rumiantes. A excepción de la paja de avena y quinua que presentaron relaciones de (A): DEPC de 42.06 y $43.05 \%$ para el flujo de $5 \% / \mathrm{h}$, respectivamente, las relaciones en el maíz chala, arveja y habas (35.74, 36.66 y $35.27 \%$, respectivamente) fueron menores al valor recomendado de $<40 \%$ (AFRC, 1995).

Con relación a la DP de la $\mathrm{PC}$, los residuos de cosecha presentaron diferencias significativas $(\mathrm{p}<0.05)$, correspondiendo a las habas y a la paja de avena 80.25 y $66.83 \%$, respectivamente. La mayor degradabilidad de la PC está relacionada, en general, con el mayor nivel de amonio y este puede contribuir al crecimiento de la población y actividad microbiana a nivel del rumen, que pueden dar lugar a un incremento en el aporte de nitrógeno microbiano al intestino delgado y maximizar el consumo de dietas altas en fibra (Ku Vera et al., 1999).
De acuerdo con el AFRC (1995), la DEPC es una estimación del total del nitrógeno capturado y utilizado por los microrganismos del rumen para la síntesis de proteína y su crecimiento. Además, la proteína de los forrajes es susceptible a la rápida degradación en el rumen, especialmente de los forrajes verdes en los cuales se degrada hasta el 73\% (Klopfenstein et al., 2001). La asociación de trébol blanco-ryegrass italiano fue superior al trébol rojo-dactylis en la DEPC en el flujo de $2 \% / \mathrm{h}$. Hecho similar ocurrió en las otras tasas de flujos (Cuadro 2), cuyos valores fueron inferiores a lo observado por Pariona (2018) en las asociaciones alfalfadactylis $(75.49 \%)$ y alfalfa-trébol-rojo (74.53\%) para la tasa de flujo de $2 \% / \mathrm{h}$.

Analizando los valores de la DEPC, se puede inferir que la asociación trébol rojodactylis sufrió la mayor influencia de las tasas de flujo, pues presentó la fracción (A) baja (11.96\%) y la fracción (B) alta (56.34\%) con relación a la asociación trébol blancorey grass italiano. Dentro de los residuos de cosecha, la quinua presentó mayores valores de (A) $(27.06 \%)$ y (C) $(7.66 \% / h)$ y bajo valor de (B) $(50.79 \%)$ con relación a las habas [(A): 20.18\%; (C): 4.66\%/h; (B): 60.07\%)]; por tanto, la quinua sufrió poco la influencia de las tasas de flujo del estudio. Por los resultados observados para la DEPC, se puede afirmar que no toda la proteína disponible de los forrajes y de los residuos de cosecha fue degradada en el rumen.

El Cuadro 2 muestra que los parámetros de la degradabilidad ruminal de la FDN de los forrajes y residuos de cosecha fueron significativamente influenciados $(p<0.05)$. $\mathrm{Si}$ se tiene en cuenta que los carbohidratos fibrosos $(\mathrm{CF}=\mathrm{FDN})$ constituyen los principales componentes de los forrajes, en el presente estudio, el trébol blanco-ryegrass italiano mostró mayores tasas de degradación (C) $(\mathrm{p}<0.05)$ al trébol rojo-dactylis. Sin embargo, estas son bajas con digestión incompleta (indegradabilidad, I: 34.92 y $29.15 \%$ de la FDN, respectivamente). Por tanto, el co- 
nocimiento de las fracciones que constituyen la FDN (D e I) es fundamental, pues se puede presumir que la fracción no digestible (I) tiene considerable efecto sobre la indigestibilidad de los alimentos (Cabral et al., 2011). La DP de la FDN (DPFDN) del trébol rojo-dactylis fue superior $(\mathrm{p}<0.05)$ a la del trébol blanco-ryegrass italiano, lo que demuestra que la pared celular del trébol rojodactylis es menos fibrosa que la del trébol blanco-ryegrass italiano, favoreciendo las condiciones al ataque microbiano $\mathrm{y}$, consecuentemente, aumentando la DPFDN.

Teniendo en consideración que el grado de digestión de la FDN es función del tiempo de permanencia del alimento en el rumen o de su velocidad de flujo de las partículas (k) (DE), el trébol blanco-ryegrass italiano y el trébol rojo-dactylis presentaron reducciones mínimas en la DEFDN (33.37 a 22.02\% y 34.57 a $22.82 \%$, respectivamente), cuando las tasas de flujo pasan de 2 a $8 \% /$ h. Estos resultados demuestran que la degradabilidad y el valor energético de la fibra se redujeron en 11.35 y 11.75 puntos porcentuales para animales de alto nivel de producción y consumo (vacas lecheras en lactancia temprana, terneros de engorde intensivo), donde el tránsito digestivo es más rápido (Salado et al., 2005). Hecho que no ha ocurrido con el trébol blanco-ryegrass italiano, pues teniendo en cuenta la DPFDN $(A+B) y k=8 \% / h$, la disminución en la degradabilidad fue de $66.16 \%$, y que asociado a la fracción (B) $(53.57 \%)$ y a la tasa de degradación (C) $(1.67 \% / \mathrm{h})$, era necesario un mayor tiempo de permanencia para degradar la pared celular (lenta degradabilidad). Por otro lado, el efecto depresor de la tasa de flujo de $8 \% / \mathrm{h}$ en la DEFDN del trébol rojo-dactylis fue de $67.79 \%$.

El maíz chala y la paja de avena presentaron superioridad en la DPFDN con relación a la arveja y la quinua $(\mathrm{p}<0.05)$. Las digestiones incompletas observadas en el maíz chala $(28.80 \%)$ y paja de avena $(26.29 \%)$ fueron menores que aquellas de la arveja $(35.20 \%)$ y quinua $(34.41 \%)$, lo que demuestra que la pared celular de los dos primeros residuos es menos fibrosa, con mayores DPFDN.

La tasa de degradación (C) varió entre 1.33 (paja de avena) y $3.67 \% / \mathrm{h}$ (quinua), siendo caracterizados como residuos de cosecha con bajas tasas de degradación ruminal para la FDN. Se han reportado tasas de degradación para la FDN del maíz chala de 2.17 y 4.77\%/h (Roa et al., 1997 y Yescas et al., 2004 , respectivamente) y para la paja de avena de $2.19-2.90 \% /$ h (Plata et al., 1994) y $0.98 \% / \mathrm{h}$ (Yescas et al., 2004). Según Mertens (1994), forrajes con valores superiores a $60 \%$ de fibra (FDN) ocasionan disminución del consumo voluntario de MS debido al efecto del hinchamiento producido en el rumen. Este fenómeno ocurrió con la arveja, presentando la menor DPFDN $(62.58 \%)$, con valor nutricional de FDN de $62.58 \%, 2.67 \% / \mathrm{h}$ de tasa de degradación e indegradabilidad de $35.20 \%$ (la más fibrosa de los residuos).

La DE, que refleja la reducción de la DP por efecto de la tasa de flujo, en el caso de los residuos de cosecha, la arveja, las habas y la quinua presentaron reducciones de la DP de $18.79,18.94$ y $18.95 \%$, respectivamente, que el maíz chala $(15.56 \%)$ y avena $(14.13 \%)$ cuando las tasas de flujo pasan de 2 a $8 \% / \mathrm{h}$. Considerando la DPFDN $=73.71 \%$ y el valor de $\mathrm{k}=8 \% / \mathrm{h}$, la disminución en la degradabilidad fue mayor en la paja de cebada $(75.01 \%)$ y que asociado a la fracción potencialmente degradable ( $\mathrm{B}=62.69 \%)$, a la tasa de degradación $(\mathrm{C}=1.33 \% / \mathrm{h}) \mathrm{y}$ fibrosidad (77.33\%), era necesario un mayor espacio de tiempo para degradar la pared celular de la paja de cebada, dando lugar a una lenta degradabilidad de la misma.

El efecto depresor de la tasa de flujo de $8 \% / \mathrm{h}$ sobre la degradabilidad de la FDN para arveja y maíz chala fueron de 62.44 y $60.98 \%$, 
respectivamente, alimentos que presentaron menores proporciones de la fracción (B) $(51.56$ y $55.59 \%)$ y tasas de degradación de 2.67 y $2.00 \% / \mathrm{h}$, respectivamente. Estos requirieron un menor tiempo de permanencia en el rumen para la degradación de las paredes celulares en relación a la paja de avena. El efecto depresor de la tasa de flujo de $8 \% / \mathrm{h}$ para la degradabilidad de la FDN en comparación a los otros residuos fueron menores en las habas (55.11\%) y quinua (48.46\%), recursos que poseen una menor proporción de las fracciones (B), tasas de degradación (C) $(57.54,58.05 \%$ y 3.00 y $3.67 \% / \mathrm{h}$, respectivamente) y menores contenidos de FDN (41.35 y $52.87 \%$, respectivamente). Por estos parámetros, las habas y la quinua desaparecieron del rumen en un menor tiempo.

\section{Agradecimiento}

Los autores agradecen al fondo de desarrollo socioeconómico del proyecto de Camisea-FOCAM por la ejecución del estudio a través del Proyecto «Generación y evaluación de un sistema computarizado en formulación de raciones al mínimo costo para ganado lechero en la provincia de Huaytara Acobamba, Tayacaja y Huancavelica de la región Huancavelica».

\section{Conclusiones}

- Se demuestra que 96 horas de incubación en rumen de muestras de forrajes y residuos de cosecha fue suficiente para alcanzar los valores máximos de la desapareción de la materia seca, proteína cruda y fibra detergente neutra.

- El comportamiento en la degradabilidad y variables ruminales indican que se puede utilizar el trébol blanco-ryegrass italiano y trébol rojo-dactylis como componentes en el balance de las raciones para rumiantes.

- El maíz chala, arveja, habas y quinua, como residuos de cosecha presentan semejanza en varios aspectos nutricionales, pudiendo utilizarse cualquiera de ellos en la alimentación de los rumiantes.

\section{Literatura Citada}

1. [AFRC] Agricultural and Food research Council. 1995. Energy and protein requirements of ruminants. Wallingford, UK: CAB International. 159 p.

2. Cabral L, Valdares S, Zervoudakis J, de Souza A, Detmann E. 2005. Degradabilidade in situ da matéria seca, da proteína cruda e fibra de alguns alimentos. Pesqui Agropecu Bras 40: 777-781.

3. Cordero FA, Contreras PJ, Curasma JC, Tunque QM, Enríquez QD. 2018. Degradabilidad y estimación del consumo de forrajes y concentrados en alpacas (Vicugna pacos). Rev Inv Vet Perú 29: 429-437. doi: 10.15381/ rivep.v29i2.14484

4. Klopfenstein TJ, Mass RA, Creighton KW, Patterson HH. 2001. Estimating forage protein degradation in the rumen. J Anim Sci 79: E208-E217.

5. Ku Vera JC, Ramírez L, Jiménez $G$, Jiménez G, Alayón JA, Ramírez, L. 1999. Árboles y arbustos para la producción animal en el trópico mexicano. En: IV Seminario Internacional sobre Sistemas Agropecuarios Sostenibles. Cali, Colombia.

6. Lagares P, Puerto J. 2001. Population and sample. Sampling techniques. Management Mathematics for European Schools. University of Sevilla. 20 p.

7. Martins AS, Zeoula LM, Prado IN, Martins EN, Loyola VR. 1999. Degradabilidade ruminal in situ da matéria seca e proteína bruta das silagens de milho e sorgo e de alguns alimentos concentrados. Rev Bras Zootecn 28: 11091117. doi: 10.1590/S1516-35981999000500029

8. Mertens DR. 1994. Regulation of forage intake. In: Forage quality, evaluation and utilization. Madison: American Society of Agronomy. p 450-493.

9. Mertens DR. 1996. Using fiber and carbohydrate analyses to formulate dairy rations. In: Informational Conference with Dairy and Forage Industries. Wisconsin, USA. 
10. Michalet-Doreau B, Ould-Bah MY. 1992. In vitro and in sacco methods for the estimation of dietary nitrogen degradability in the rumen: a review. Anim Feed Sci Tech 40: 57-86. doi: 10.1016/0377-8401(92)90112-J

11. Molina LR, Rodriguez NM, de Sousa B, Goncalves L, Borges I. 2003. Parâmetros de degradabilidad potencial da materia seca e da proteína bruta das silagens de seis genotipos de sorgo (Sorghum bicolor (L.) Moench), com e sem tanino no grão, avaliados pela técnica in situ. Rev Bras Zootecn 32: 222228.doi:10.1590/S1516-35982003000100028.

12. Orskov ER, Mcdonald I. 1979. The estimation of protein degradability in the rumen from incubation measurements weighted according to rate of passage. J Agri Sci 92: 499-453. doi: 10.1017/ S0021859600063048

13. Pariona L. 2018. Composición química y cinética de la degradabilidad ruminal de forrajes y concentrados en bovinos en la Universidad Nacional de Huancavelica. Tesis de Ingeniero Zootecnista. Huancavelica, Perú: Univ. Nacional de Huancavelica. $84 \mathrm{p}$.

14. Plata PF, Mendoza GD, BárcenaGama JR, González S. 1994. Effect of a yeast culture (Saccharomyces cerevisiae) on neutral detergent fiber digestion in steers fed oat straw based diets. Anim Feed Sci Tech 49: 203-210. doi: 10.1016/0377-8401(94)90046-9

15. Roa ML, Bárcena-Gama JR, Gonzalez, S, Mendoza G, Ortega ME, García C. 1997. Effect of fiber source and a yeast culture (Saccharomyces cerevisiae) on digestion and the environment in the rumen cattle. Anim Feed Sci Tech 64: 327-336. doi: 10.1016/S03778401(96)01015-2

16. Salado EE, Comeron EA, Silva C, Gaggiotti MC, Alesso A, Pardo J. 2005. Cascarilla de soja y afrechillo de trigo: cinética de la degradabilidad ruminal de la fibra. En: XIX Reunión de la Asociación Latinoamericana de Producción Animal. Tampico, México.

17. Silva DJ, Quiroz AC. 2002. Análise de alimentos: métodos químicos y biológicos. Minas Gerais, Brasil: Universidade Federal de Viçosa. $235 \mathrm{p}$.

18. Van Soest PJ. 1994. Nutritional ecology of the ruminant. $2^{\text {nd }}$ ed. Ithaca, New York: Cornell University Press. $476 \mathrm{p}$.

19. Yescas $R$, Bárcena-Gama R, Mendoza GD, González SS, Cobos M, Ortega ME. 2004. Digestibilidad in situ de dietas con rastrojo de maíz o paja de avena con enzimas fibrolíticas. Agrociencia $38: 23-31$. 